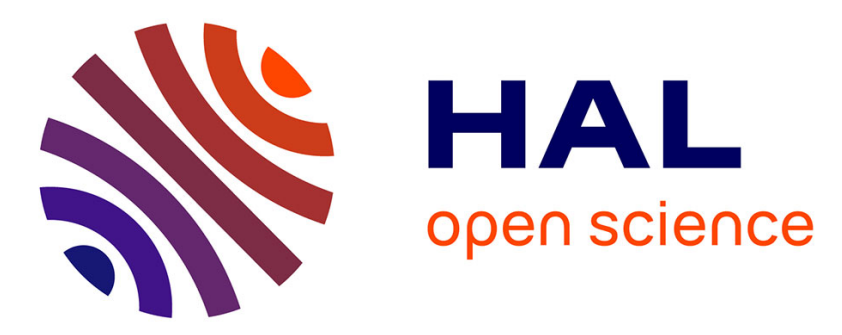

\title{
Innovations budgétaires en PME : l'influence du secteur d'activité et du profil du dirigeant
}

Zouhour Ben Hamadi, Philippe Chapellier, Fabienne Villesèque-Dubus

\section{To cite this version:}

Zouhour Ben Hamadi, Philippe Chapellier, Fabienne Villesèque-Dubus. Innovations budgétaires en PME : l'influence du secteur d'activité et du profil du dirigeant. Innovations - Revue d'économie et de management de l'innovation, 2014, 43 (1), pp.223. 10.3917/inno.043.0223 . hal-02010369

\section{HAL Id: hal-02010369 \\ https://hal.science/hal-02010369}

Submitted on 14 Jan 2020

HAL is a multi-disciplinary open access archive for the deposit and dissemination of scientific research documents, whether they are published or not. The documents may come from teaching and research institutions in France or abroad, or from public or private research centers.
L'archive ouverte pluridisciplinaire HAL, est destinée au dépôt et à la diffusion de documents scientifiques de niveau recherche, publiés ou non, émanant des établissements d'enseignement et de recherche français ou étrangers, des laboratoires publics ou privés. 


\title{
Innovations budgétaires en PME : l'influence du secteur d'activité et du profil du dirigeant
}

\section{Budgetary innovations in SMEs: the influence of the activity sectors and profile of leaders}

\author{
Zouhour Ben Hamadi \\ Doctorante en Sc. de Gestion \\ Montpellier Recherche \\ en Management \\ Labex Entreprendre \\ zouhourhamadi@yahoo.fr
}

\author{
Philippe Chapellier \\ MCF HDR en Sc. de gestion \\ Montpellier Recherche \\ en Management \\ Labex Entreprendre \\ philippe.chapellier@univ-montp2.fr
}

\author{
Fabienne Villesèque-Dubus \\ MCF HDR en Sc. de gestion \\ Montpellier Recherche \\ en Management \\ Labex Entreprendre \\ fabienne.villeseque@univ-perp.fr
}

\section{Résumé}

L'objectif de cette étude est de déterminer dans quelle mesure le secteur d'activité d'une part et le profil des dirigeants d'autre part, influencent l'adoption d'innovations budgétaires en PME.

Une enquête par questionnaire administrée en face à face a été réalisée auprès de 101 dirigeants de PME industrielles tunisiennes.

Le profil des dirigeants d'une part, et le secteur d'activité d'autre part, apparaissent comme des variables clés liées à l'adoption d'innovations budgétaires dans les PME.

L'étude montre ainsi que la diversité des contextes et des acteurs oblige les institutions, organisations et réseaux composant les systèmes sectoriels de l'innovation (SSI) à tenir compte des spécificités sectorielles et individuelles pour favoriser la production de connaissances nouvelles utiles à l'innovation.

Elle souligne aussi l'isomorphisme des pratiques innovantes des dirigeants aux perceptions et profils similaires.

Mots clés : innovations budgétaires, contingences, secteurs d'activité, profils de dirigeants, PME Codes JEL : L26, M41

\begin{abstract}
This work aims to determine to what extent activity sector and manager profile influence the adoption of budgetary innovations in SMEs.

101 managers of Tunisian industrial SMEs were interviewed, using a face-to-face questionnaire. The manager profile on the one hand, and the activity sector on the other hand, prove to be key variables with respect to the adoption of budgetary innovations in SMEs.

Our study shows that the diversity of contexts and actors requires the institutions, organizations, and networks composing the sectoral systems of innovation (SSI) to take into account sectoral and individual specificities, in order to promote the production of new knowledge useful to budgetary innovation.

The study also stresses the isomorphism of innovative practices among the managers with similar profiles and perceptions.
\end{abstract}

Keywords: budgetary innovations, contingencies, activity sectors, manager profiles, SMEs

Codes JEL: L26, M41 


\section{Introduction}

La typologie la plus fréquemment proposée dans la littérature en sciences de gestion sur les innovations oppose l'innovation technologique à l'innovation managériale (Jaouen et Le Roy, 2013). L'élément distinctif serait le domaine de l'organisation affecté par l'innovation : l'innovation technologique affecterait les caractéristiques physiques et matérielles des biens, des services, ou des procédés, alors que l'innovation managériale affecterait les modes de coordination et de motivation des éléments de la structure sociale qui en assurent le fonctionnement (Alcouffe, 2006). La littérature sur l'innovation technologique est abondante (Abernathy et Utterback, 1978 ; Henderson et Clark, 1990 ; Teece et al., 1997, Govindarajan et Trimble, 2012). Comparativement, la littérature sur l'innovation managériale, bien qu'elle se soit sensiblement enrichie au cours des dix dernières années, reste relativement réduite (Le Roy et al, 2012). Jaouen et Le Roy (2013, p.1) soulignent ainsi que «les entreprises, les managers, les pouvoirs publics, les chercheurs en économie et en management ont jusqu'à présent consacré l'essentiel de leur attention à l'innovation technologique au détriment de l'innovation managériale ». Birkinshaw et al (2008) expliquent que l'importance de ce concept a été largement sous-estimée en comparaison avec celui d'innovation technologique. Hamel (2007) considère l'innovation managériale comme le «parent pauvre» de la recherche sur les innovations.

De nombreux auteurs (Hamel, 2006, 2007 ; Damanpour et Aravid, 2011 ; Mol et Birkinshaw, 2009) soulignent ainsi le besoin de mieux apprécier cette forme d'innovation qui pourrait davantage assurer la pérennité des entreprises que les autres, plus traditionnelles, comme l'innovation dans les produits ou les procédés. Pour Birkinshaw, Hamel et Mol (2008), fondateurs de l'Innovation Management Lab à la London Business School, l'innovation managériale est le principal facteur explicatif des performances de l'entreprise. Pour Mol et Birkinshaw (2009), ce type d'innovation modifie la façon dont l'ensemble des managers cherche à atteindre les objectifs de performance. Ils notent que «dans de nombreux cas, l'innovation managériale est la pièce manquante du puzzle de l'innovation » (Mol et Birkinshaw, 2006, p.26).

Notre article s'intéresse à la question des innovations managériales en matière de contrôle de gestion, et plus précisément en matière budgétaire, dans le contexte spécifique de la PME. Cette question est intéressante en raison tout d'abord de la spécificité des PME (Marchesnay, 1997 ; Julien, 1997) et des systèmes de contrôle qui s'y exercent (Nobre, 2001 ; Chapellier et Dupuy, 2013). Cette spécificité influerait sur leur aptitude à innover (Hausman, 2005). Naro (2006) souligne notamment la difficulté de concilier l'innovation (perçue comme une démarche complexe) avec les besoins de simplicité et de flexibilité spécifiques aux PME. 
Cette question est intéressante ensuite en raison des constantes discussions et débats relatifs à l'utilité et à l'utilisation du budget qui posent la question de son renouvellement en tant qu'innovation managériale. A cet égard, si le recours à l'outil budgétaire dans les organisations n'est pas neuf (selon Berland (1999), il daterait en France du début des années 30), il demeure toujours d'actualité et largement présent dans les organisations. Les nombreuses études, enquêtes et thèses réalisées sur le sujet ces dernières années soulignent les multiples évolutions quant à leurs modes de conception (par activité, par projets, budget base zéro, budgets environnementaux...) ou d'utilisation (interactive, à des fins de communication externe...), laissant légitimement penser que les budgets sont l'objet d'innovations (DFCG, 1994 ; Jordan, 1998 ; Berland, 1999 ; Villesèque, 2003 ; Sponem, 2004 ; Mousli, 2007 ; Nguyen Tan Hon, 2008).

A l'instar des travaux de Malerba (2005), notre étude interroge la nécessité pour les institutions, organisations et réseaux composant les systèmes sectoriels de l'innovation (SSI), de tenir compte des spécificités sectorielles et individuelles des PME pour favoriser la production de connaissances nouvelles utiles à l'innovation budgétaire en PME.

Deux objectifs sont attachés à cette étude. Le premier est d'interroger les spécificités et convergences sectorielles de l'innovation budgétaire dans les PME. La question de recherche attachée à cette problématique est la suivante : dans quelle mesure le secteur d'activité dans lequel opère la PME influence-t-il l'adoption d'innovations budgétaires ?

Les travaux réalisés sur le thème du dirigeant de PME (Marchesnay, 1991 ; Julien et Marchesnay, 1996) ont par ailleurs montré l'influence que peuvent avoir ses caractéristiques personnelles sur la gestion de son entreprise : sur l'élaboration de la stratégie (Fonrouge, 2002 ; Kotey et Meredith, 1997), sur les pratiques en matière de comptabilité de gestion (Saboly, 1994 ; Chapellier, 2011) ou sur les objectifs ou les caractéristiques du système de données comptables (Lavigne , 2002 ; Affès et Chabchoub, 2007) par exemple. Le second objectif de la recherche est de déterminer dans quelle mesure le profil du dirigeant influence l'adoption d'innovations budgétaires dans les PME.

Après avoir présenté le cadre conceptuel et les hypothèses de la recherche, nous exposons la méthodologie mise en œuvre et les résultats obtenus. Ceux-ci sont ensuite discutés dans une dernière partie, au regard notamment du concept de SSI, adapté pour l'occasion à des innovations managériales. 


\section{Cadre conceptuel et hypothèses de la recherche}

Nous précisons tout d'abord les définitions d'une innovation managériale et d'une innovation budgétaire retenues dans cette étude. Nous parcourons ensuite la littérature sur l'impact du secteur d'activité, du profil des dirigeants et d'autres facteurs d'influence, sur l'adoption d'innovations managériales. Nous présentons enfin les hypothèses et le modèle de recherche.

\subsection{Concepts d'innovation managériale et budgétaire}

\subsubsection{Du concept d'innovation managériale...}

Birkinshaw, Hamel et Mol (2008, p.825) définissent l'innovation managériale comme « l'invention et la mise en cuvre d'une pratique, d'un processus, d'une structure ou d'une technique de management nouvelles au vu de l'état de l'art, et qui contribuent à l'atteinte des objectifs organisationnels». Ils ajoutent dans le même article que « dans son sens le plus large, l'innovation managériale peut être définie comme une différence dans la forme, la qualité ou l'état des activités managériales au fil du temps dans une organisation ».

Le Roy et al (2012) notent que cette définition recouvre deux acceptions possibles du concept :

- l'innovation managériale peut être considérée comme l'adoption par une organisation d'une pratique ou d'une méthode de management complètement nouvelle par rapport aux pratiques et méthodes de management connues: l'organisation met au point une innovation et la met en œuvre la première,

- l'innovation managériale peut être considérée comme l'adoption par une entreprise d'une pratique ou d'une méthode de management qui existe déjà mais qui est nouvelle par rapport à ses pratiques et méthodes de management actuelles : l'organisation ne met pas au point l'innovation et n'est pas nécessairement la première à l'adopter.

C'est la deuxième acception que nous retenons dans cette recherche. Nous définissons ainsi l'innovation managériale comme l'adoption par une organisation d'une méthode ou d'une pratique de management qui représente un éloignement perçu comme significatif de l'état du management dans cette organisation au moment où il apparaît pour la première fois.

Nous rejoignons ainsi Alcouffe et al (2003, p.8) qui expliquent que «le caractère nouveau d'une innovation s'entend par rapport à la personne ou au groupe de personne qui considère son adoption. Dans ce sens, un outil de gestion sera une innovation s'il est perçu comme nouveau par les acteurs ». Cette définition n'implique pas de caractère concret et ouvre la voie aux innovations relatives (Martineau, 2008). L'accent est dès lors mis sur la perception (Godowski, 2003) : il suffit qu'un individu considère une pratique ou une méthode comme nouvelle pour qu'elle soit considérée comme une innovation. 


\subsection{2 ...au concept d'innovation budgétaire}

Les budgets ont fait l'objet, au cours du dernier siècle, d'innovations. Il est possible, à partir d'une lecture « historique » des outils de gestion, de contrôle, ou de pilotage budgétaire, de reconstituer l'apparition de changements dans les modes de conception et/ou d'utilisation des budgets. Ainsi, qu'elles soient explicitées en tant que telles ou non, les innovations en matière de budgets sont nombreuses. Les recherches historiques conduites par Berland à partir de 1999 montrent tout d'abord que le budget est apparu aux Etats-Unis avec la parution de l'ouvrage de McKinsey en 1922, puis en France quelques années plus tard, constituant à cette époque-là une innovation managériale. Le contrôle budgétaire constitue alors le thème affiché de la conférence de Genève en 1930 organisée par l'IIOST (Institut International de l'Organisation Scientifique du Travail). Cette date marque le début d'un processus d'institutionnalisation du budget, soutenu par une idéologie managériale forte et par de nombreux acteurs-clés dans le processus de diffusion de l'outil (Alcouffe et al, 2003 ; Berland, 1999, 2002 ; Berland et Chiapello, 2009). Le budget se serait en particulier diffusé grâce à l'action de certains acteurs jouant le rôle d'acteurs réseaux, accompagnée notamment d'une certaine rhétorique autour de l'outil, facilitant sa diffusion (Alcouffe et al., 2008 ; Berland et Chiapello, 2009).

Les budgets ont depuis leur apparition dans les organisations fait l'objet de nombreuses et parfois profondes mutations en premier lieu dans leurs modes de conception.

L'apparition du Budget Base Zéro (BBZ), dont Texas Instrument a été l'un des pionniers dans la mise en place (Pyrrh, 1976), constitue un exemple d'innovation budgétaire. Le BBZ, présenté comme particulièrement adapté à la prévision des frais généraux, permet alors de prévoir en repartant de zéro, bousculant à cette époque les principes de prévision fondés sur une approche passéiste de l'entreprise, et basés sur la reconduction budgétaire des dépenses.

Ont ensuite suivi d'autres types d'innovations budgétaires, centrés sur l' « abandon » du budget en réponse aux nombreuses critiques à son égard. L'enquête conduite par la $\mathrm{DFCG}^{1}$ en 1994 intitulée «Faut-il tuer le budget?», les travaux du CAM- $\mathrm{I}^{2}$ et du «Beyond Budgeting Roundtable » aux Etats-Unis (Schmidt, 1992 ; Banham, 2000 ; Hope et Fraser, 1997, 1999) ou encore ceux sur les propositions de gestion sans budget en France (Berland, 2002, 2004) en témoignent.

Parallèlement à ces questions sont apparues les démarches dites d'Activity Based Budgeting (Cooper et Slagmulder, 2000 ; Brimson et Antos, 1999) pouvant également être perçues comme des innovations budgétaires. Ce type de démarche, qui nécessite d'adopter des maillages par

\footnotetext{
${ }^{1}$ Association Nationale des Directeurs Financiers et de Contrôle de Gestion

${ }^{2}$ Consortium for Advanced Management International
} 
activités ou processus, se fonde sur une vision transversale et décloisonnée de l'entreprise, éloignée des modèles hiérarchico-fonctionnels classiques. Ainsi, l'établissement de budgets transversaux organisés par activités, processus ou projets (Dupuy et Villesèque, 2003 ; Villesèque-Dubus, 2005) constituent un changement certain dans les organisations, lié à une démarche de budgétisation par activités et non par ressources, de gestion d'inducteurs de coûts plutôt que d'unités d'œuvre. Ils entrainent des changements organisationnels dans la mise en place de nouvelles logiques liées à l'attribution des responsabilités.

Les budgets continuent aujourd'hui à évoluer dans leurs modes de conception, en réponse également à des besoins sociétaux. On assiste ainsi à l'émergence de budgets environnementaux (Desmazes et Lafontaine, 2007), inscrits dans une logique de responsabilité sociale de l'entreprise. Ces budgets permettraient d'une part de prévoir les dépenses environnementales, d'autre part de suivre et retracer les actions en matière de développement durable.

Au-delà de ces nouvelles formes budgétaires, vues comme des innovations managériales, l'innovation budgétaire peut aussi se concrétiser dans la façon d'utiliser le budget. Ainsi, si conformément à ses attributions classiques le budget a pour mission de coordonner et de communiquer les actions, de prévoir et de déléguer (Bouquin, 1998), il peut être aussi utilisé comme un outil interactif au sens de Simons (1995), du fait des changements induits dans les modes de pilotage, de sa capacité à faciliter l'émergence de la stratégie et l'apprentissage, et à nourrir le dialogue et les échanges. Son usage en ce sens constitue une innovation managériale.

Les budgets ont ainsi fait l'objet, au cours du dernier siècle, d'innovations. En lien avec la définition de l'innovation managériale retenue dans cette étude, nous définissons l'innovation budgétaire comme l'adoption par une organisation d'une méthode ou d'une pratique budgétaire, qui représente un éloignement perçu comme significatif de l'état du management dans cette organisation au moment où il apparaît pour la première fois.

\subsection{L'impact du secteur d'activité sur l'adoption d'innovations budgétaires en PME}

«Les processus d'innovation varient considérablement d'un secteur à l'autre ... Certains secteurs se caractérisent par une évolution rapide et des innovations radicales alors que d'autres connaissent une évolution plus modeste et plus progressive»(Manuel d'Oslo, 2005, p.44). Malerba (2005) explique cet état de fait par l'existence de différences d'un secteur à l'autre du point de vue du rythme du progrès, de l'accès au savoir, des facteurs institutionnels et des structures organisationnelles.

La plupart des recherches en sciences de gestion réalisées sur le thème de l'adoption d'innovations managériales dans les organisations, étudient les conditions d'adoption des innovations dans un secteur d'activité donné. Nobre et Biron (2002) par exemple décrivent une 
expérimentation de l'adoption de la méthode $\mathrm{ABC}$ dans le secteur hospitalier. Dhifallah et al (2008) s'intéressent à l'adoption d'innovations dans des entreprises industrielles de fabrication de matériaux. Ferrary (2008) étudie l'adoption d'innovations dans des start-up de hautes technologies alors que Simon et Tellier (2008) observent l'émergence de projets créatifs chez des fabricants de semi-conducteurs.

Des congrès et colloques ont eu pour objectif d'interroger les spécificités et convergences sectorielles de l'innovation en confrontant des travaux réalisés sur ce thème dans différents secteurs d'activité. Mais les études incluant au modèle de recherche le secteur d'activité en vue de mettre en évidence l'importance d'éléments propres aux différents secteurs sur l'adoption d'innovations managériales restent rares. On peut citer à cet égard celle de Brion et al. (2008) qui s'intéresse aux facteurs favorisant différents types d'innovations dans 307 PME opérant dans l'industrie, le commerce, la construction et le service, ou encore celle de Gadille et d'Iribarne (2000) qui montre l'existence de différences significatives en matière d'adoption d'innovations technologiques selon le secteur d'activité. Mais ces dernières ne concernent pas spécifiquement les innovations managériales.

Les spécificités sectorielles influencent-elles l'adoption d'innovations budgétaires dans les PME ? Pour répondre à cette question, nous proposons de tester l'hypothèse selon laquelle :

\section{H1 : il existe une association entre le secteur d'activité et l'adoption d'innovations budgétaires en PME.}

\subsection{L'impact du profil du dirigeant sur l'adoption d'innovations budgétaires en PME}

Plusieurs recherches soulignent l'importance du profil des acteurs dans l'émergence d'innovations managériales dans les organisations (Khan et Manopichetwattana, 1989a, 1989b ; Alcouffe et al, 2003). Pour Boldrini (2008, p.16), «la personnalité du dirigeant de l'entreprise, souvent propriétaire, est décisive dans le succès des innovations... Comme il centralise la prise de décision, la capacité d'innovation ultérieure de l'entreprise dépend beaucoup de lui ».

Ces auteurs soutiennent que les individus sont actifs par rapport à la situation et non contraints par cette dernière. Ainsi, et plus précisément, ils soulignent que si les caractéristiques organisationnelles et de contexte peuvent, dans une certaine mesure, limiter et déterminer les choix de l'acteur, ce dernier n'en reste pas moins libre et doué d'intentionnalité. Il dispose d'une marge de liberté (Crozier et Friedberg, 1977). En ce sens, Lorino (1995) souligne l'importance de la prise en considération de l'autonomie cognitive des acteurs : chacun détient une part de la connaissance nécessaire à l'action.

Shields et Young (1993) notent ainsi que le succès de l'implantation d'un système de comptabilité de gestion est plus déterminé par des facteurs comportementaux que par des facteurs 
économiques et techniques. Solle et Rouby (2003) expliquent que l'émergence d'outils de gestion est intimement liée aux relations sociales. Moisdon (1997) déclare que rationnaliser le choix des outils de gestion est une vision réductrice et que ce choix dépend largement d'appréciations subjectives. Walley et al. (1994) relèvent sept facteurs déterminants ayant une influence significative sur l'adoption des systèmes « modernes » de calcul de coûts. Parmi ces sept facteurs, cinq se rattachent aux caractéristiques des acteurs.

Les facteurs comportementaux retenus par la littérature sur les PME sont le plus souvent organisés autour de l'acteur central de ce type d'entreprises : son dirigeant. Les travaux dans ce domaine soulignent alors que le profil du dirigeant joue un rôle très particulier qui fait la spécificité de ce type d'organisation : «comprendre la PME, c'est tout d'abord découvrir le profil et percer les motivations de son propriétaire-dirigeant » (Raymond et al., 2004, p.54). Ses caractéristiques pourraient avoir un impact significatif sur l'adoption d'innovations budgétaires et à ce titre, méritent d'être analysées.

Tout comme Khan et Manopichetwattana (1989a, 1989b) qui ont tenté d'identifier les facteurs de contingence relatifs au profil des dirigeants expliquant les différences entre PME innovantes et PME non-innovantes, nous essayerons de déterminer dans quelle mesure le profil des dirigeants influence l'adoption d'innovations budgétaires en PME.

Nous établirons pour cela une analyse typologique dans un contexte au sein duquel, à notre connaissance, cela n'a jamais été réalisé (la PME tunisienne), afin de pouvoir ensuite rapprocher les profils de dirigeants identifiés, des innovations budgétaires adoptées.

A la lecture des travaux réalisés, les caractéristiques descriptives du profil du dirigeant retenues dans notre étude sont son âge (Baldrige et Burnham, 1975 ; Chapellier, 1996), son niveau et son type de formation (Kessler et Chakrabarti, 1996, Mohammed, 2011), son expérience (Damanpour et Schneider, 2006), les buts qu'il poursuit (Julien et Marchesnay, 1996), son aversion au risque (Hofstede, 2001 ; West, 1990 ; Saleh et Wang, 1993) et son orientation court ou long terme ${ }^{3}$ (Hofstede, 2001 ; Brion et al, 2008).

Nous proposons de tester l'hypothèse selon laquelle :

\section{H2 : Il existe une association entre le profil du dirigeant et l'adoption d'innovations budgétaires en PME.}

\footnotetext{
${ }^{3}$ Hofstede (2001) associe l'orientation à court-terme au respect de valeurs orientées vers le passé et le présent (comme respecter les traditions et satisfaire ses engagements sociaux), et l'orientation long terme au respect de valeurs orientées vers le futur (comme faire des économies et être persévérant).
} 


\subsection{Autres facteurs d'influence de l'adoption d'innovations budgétaires en PME}

Nous testerons par ailleurs l'influence de facteurs contextuels et structurels sur l'adoption d'innovations budgétaires. Pour cela seront testés les liens entre l'adoption en PME d'innovations budgétaires et quatre variables de contrôle identifiées par différents auteurs comme susceptibles d'influencer les pratiques de gestion en PME.

\section{- La taille de l'entreprise}

Zahra et al. (2000) et Damanpour et Schneider (2006) montrent que les entreprises les plus grandes sont les plus innovantes. Les entreprises les plus grandes pourraient tout d'abord plus facilement que les petites répartir les risques d'échec et absorber les coûts de l'innovation. Elles auraient ensuite une meilleure capacité pour établir et entretenir des installations scientifiques et pour investir dans l'innovation. Elles disposeraient enfin de ressources plus importantes pour embaucher du personnel qualifié. Zmud et Link (1984), quant à eux, trouvent une relation négative entre la taille des entreprises et l'adoption d'innovations.

L'adoption d'innovations budgétaires des PME les plus grandes diffère-t-elle de celle des PME les plus petites? Pour répondre à cette question, nous proposons d'intégrer la taille de l'entreprise comme variable de contrôle.

\section{- L'âge de l'entreprise}

Plusieurs études ont montré l'existence de liens entre l'âge de l'entreprise et ses pratiques de gestion (Holmes et Nicholls, 1989; Chapellier, 1996). L'idée avancée par Mintzberg (1982) et Dupuy et al. (1989) est que l'âge de l'entreprise fait inévitablement référence à son passé, à son histoire et que les événements qui ont marqué cette histoire (changement de propriétaire, difficultés) influent très directement et spécifiquement sur les pratiques de gestion. L'adoption d'innovations budgétaires des PME les plus anciennes diffère-t-elle de celle des plus jeunes ? Aucune recherche à notre connaissance ne s'est intéressée à cette question. Nous proposons de ce fait d'intégrer l'âge des PME comme variable de contrôle.

\section{- La structure de propriété}

Une entreprise est dite familiale lorsque le dirigeant dispose, seul ou avec les membres de sa famille proche, à la fois du pouvoir de gestion et de la propriété du capital. Bescos et Mendoza (1998) et Cheffi et Nekhili (2011) notent que la présence dans une entreprise d'associés ou d'actionnaires non apparentés conduit le dirigeant à mettre en place des outils comptables formels pour légitimer ses actions et ses décisions, et pour réduire l'asymétrie informationnelle perçue par les associés ou actionnaires extérieurs. Germain (2001) note que le mode de propriété de l'entreprise est à l'origine de différences dans l'usage des tableaux de bord. Lavigne et SaintPierre (2002) expliquent que la présence d'actionnaires ne faisant pas partie de la famille du 
dirigeant incite ce dernier à une plus grande formalisation dans la préparation des données financières afin d'atténuer les problèmes d'agence. La littérature montre donc que la structure de propriété influe sur les pratiques de gestion. Aucune recherche à notre connaissance ne s'est intéressée à la question du lien entre la structure de propriété et l'adoption d'innovations budgétaires en PME. Nous proposons d'intégrer la structure de propriété des PME comme variable de contrôle.

\section{- L'incertitude perçue de l'environnement}

Plusieurs auteurs avancent que l'incertitude perçue par le dirigeant de l'environnement dans lequel opère son entreprise, influence les caractéristiques du système de gestion (Chong et Chong, 1997 ; Haldma et Lääts, 2002 ; Germain, 2004 ; Bescos et al., 2004 ; Abdel-Kader et Luther, 2008). Germain (2004) montre par exemple que les PME dont l'environnement est perçu par leur dirigeant comme dynamique disposent de tableaux de bord plus complexes. Huet (2006) indique que l'innovation peut apparaître en réponse à des modifications environnementales rendant partiellement obsolètes les connaissances disponibles. Bergeron (1996) en revanche n'identifie pas de liens significatifs entre l'incertitude perçue de l'environnement et la complexité des systèmes de coûts et budgétaires. L'adoption d'innovations budgétaires des PME dont l'environnement est perçu comme incertain diffère-t-elle de celle des PME où l'environnement est perçu comme plus stable? Pour répondre à cette question, nous proposons d'intégrer l'incertitude perçue de l'environnement comme variable de contrôle.

Le modèle de recherche peut donc être représenté ainsi :

Figure 1 : Le modèle de recherche

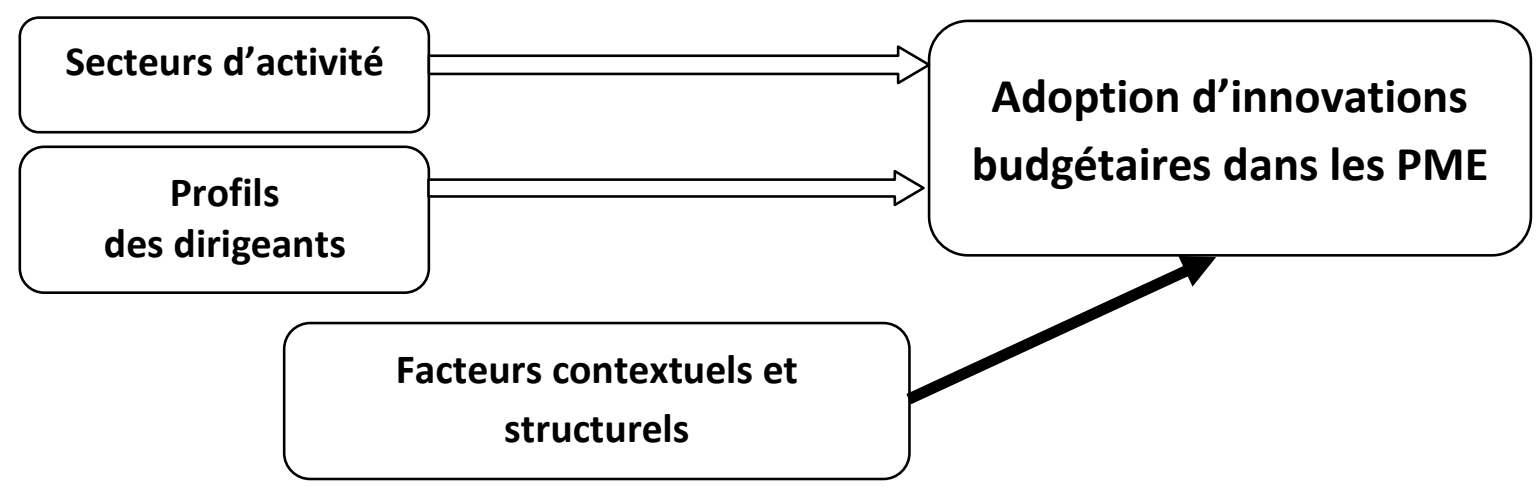

\section{Méthodologie de recherche}

La méthode de recueil des données, la manière dont les variables ont été opérationnalisées, les caractéristiques de l'échantillon et la méthode d'analyse des données, sont successivement présentées. 


\subsection{La méthode de recueil des données}

L'enquête s'est déroulée en deux temps. Tout d'abord une enquête préliminaire a été conduite par administration en face-à-face d'une première version du questionnaire. Ce pré-test, réalisé en novembre et décembre 2010 auprès de 25 dirigeants de PME tunisiennes, a permis une mise à l'épreuve de la forme des questions, de leur ordonnancement, une revue du vocabulaire courant, une vérification de la compréhension des répondants et de la pertinence des modalités de réponse proposées.

L'administration en face à face de la version finale du questionnaire a été réalisée du 20 février au 5 mai 2011. Compte tenu des contraintes liées au mode de collecte des données en face-à-face, l'étude a été réalisée sur une surface géographique couvrant trois gouvernorats parmi les plus industrialisés de Tunisie (Sousse, Monastir et Mahdia). La population ciblée était de 445 PME industrielles. Un tirage aléatoire de 282 PME a été réalisé, 101 dirigeants ont été interrogés, soit un taux de réponse de 35,8\%.

Les évènements politiques qu'a connus la Tunisie au début de l'année 2011 ont rendu la collecte des données difficile. Mais un mode d'administration du questionnaire en face-à-face s'imposait à notre sens car il autorise un recueil d'informations plus complet, plus détaillé et de meilleure qualité que l'enquête postale ou électronique (Evrard et $a l ., 2009$ ). Il a permis d'apporter certaines précisions utiles au répondant et à la validité de l'étude grâce à la possibilité de reformuler, de clarifier et d'expliquer si nécessaire les questions difficiles ou mal comprises (Thiétart, 2007). De plus, la confidentialité qui entoure la gestion comptable en PME aurait probablement conduit la plupart des dirigeants à refuser de répondre à un questionnaire reçu par voie postale ou électronique, alors que l'administration en face à face a généré de la confiance et rendu possible la réalisation de cette recherche.

Cette méthode n'est toutefois pas sans limite, la principale étant que les données recueillies sont auto-rapportées et de l'ordre de la perception, et que le dirigeant peut être conduit à répondre pour se valoriser ou pour se conformer aux normes sociales (Evrard et al., 2009).

\subsection{L'opérationnalisation des variables}

Le questionnaire était structuré en trois thématiques : les caractéristiques de l'entreprise et de son environnement, les innovations budgétaires adoptées au cours des cinq dernières années et les caractéristiques du dirigeant de PME. Il contenait à la fois des questions fermées avec échelles graduées et des questions ouvertes qui permettent de donner des réponses plus vastes.

Le tableau 1 présente la manière dont les variables ont été opérationnalisées. 
Tableau 1 : Opérationnalisation des variables

\begin{tabular}{|l|l|l|}
\hline \multicolumn{3}{|c|}{ Facteurs socioprofessionnels du dirigeant } \\
\hline 1. Age du dirigeant & V. Multinomiale & $\begin{array}{l}\text { Prend une valeur comprise entre 1 et 5 selon la tranche d'âge } \\
\text { du dirigeant : moins de 35 ans ; 36 à 45 ans ; 46 à 55 ans ; 56 } \\
\text { à } 65 \text { ans ; plus de 65 ans }\end{array}$ \\
\hline $\begin{array}{l}\text { 2. Niveau de } \\
\text { formation du } \\
\text { dirigeant }\end{array}$ & V. Multinomiale & $\begin{array}{l}\text { Prend une valeur comprise entre 1 et 6, du dirigeant } \\
\text { autodidacte au dirigeant diplômé de niveau minimum bac+5 }\end{array}$ \\
\hline $\begin{array}{l}\text { 3. Type de formation } \\
\text { du dirigeant }\end{array}$ & V. Multinomiale & $\begin{array}{l}\text { Formation en gestion/comptabilité } \\
\text { Formation dans une autre discipline } \\
\text { Sans formation }\end{array}$ \\
\hline $\begin{array}{l}\text { 4. Expérience du } \\
\text { dirigeant }\end{array}$ & V. Multinomiale & $\begin{array}{l}\text { Moins de } 5 \text { ans } \\
\text { Entre 5 et } 10 \text { ans } \\
\text { Plus de } 10 \text { ans }\end{array}$ \\
\hline $\begin{array}{l}\text { 5. But principal du } \\
\text { dirigeant }\end{array}$ & V. Multinomiale & $\begin{array}{l}\text { Prend une valeur entre 1 et 4 selon le principal but visé par le } \\
\text { dirigeant (pérennité, indépendance, croissance ou profit) }\end{array}$ \\
\hline
\end{tabular}

\begin{tabular}{|l|l|l|}
\hline \multicolumn{2}{|c|}{ Facteurs culturels du dirigeant } \\
\hline 6. Aversion au risque & V. Métriques & $\begin{array}{l}\text { Mesurée par 6 items (échelle de Likert de 5 points) repris de } \\
\text { la recherche de Hofstede (2001) }\end{array}$ \\
\hline $\begin{array}{l}\text { 7. Orientation Long } \\
\text { terme / Court terme }\end{array}$ & V. Métriques & $\begin{array}{l}\text { Mesurée par 6 items (échelle de Likert de 5 points) repris de } \\
\text { la recherche de Hofstede (2001) }\end{array}$ \\
\hline
\end{tabular}

\begin{tabular}{|l|l|}
\hline \multicolumn{2}{|c|}{ Secteurs d'activité } \\
\hline Variable multinomiale & Entreprises agroalimentaires \\
& Entreprises de l'industrie lourde (construction métallique, \\
& traitements des métaux, équipements automobiles...) \\
& Entreprises manufacturières (textile et habillement) \\
\hline
\end{tabular}

\begin{tabular}{|l|l|}
\hline \multicolumn{2}{|c|}{ Variable à expliquer : L'adoption d'innovations budgétaires } \\
\hline Variable dichotomique & $\begin{array}{l}\text { 1. Entreprise qui a réalisé au moins une innovation } \\
\text { budgétaire au cours des 5 dernières années } \\
\text { 0. Entreprise qui n'a pas réalisé d'innovations budgétaires au } \\
\text { cours des } 5 \text { dernières années }\end{array}$ \\
\hline $\begin{array}{l}\text { Les différentes innovations budgétaires citées par les dirigeants : } \\
\text { La diversité des budgets mis en œuvre a significativement augmenté } \\
\text { Le degré de détail dans les budgets est beaucoup plus important } \\
\text { L'implication des dirigeants dans le suivi budgétaire s'est considérablement accrue, le mode de suivi a } \\
\text { changé } \\
\text { Le système budgétaire est nouvellement instauré dans l'entreprise } \\
\text { Le passage vers un budget à Base Zéro } \\
\text { Le passage vers un budget ventilé par activité } \\
\text { Le passage vers un budget ventilé par projet }\end{array}$ \\
\hline \hline
\end{tabular}

\begin{tabular}{|l|l|l|}
\hline \hline \multicolumn{2}{|c|}{ Variables de contrôle } \\
\hline Taille de l'entreprise & V. Multinomiale & $\begin{array}{l}\text { Entre } 10 \text { et } 50 \text { employés } \\
\text { Entre 51 et } 100 \text { employés } \\
\text { Entre 101 et 300 employés }\end{array}$ \\
\hline Age de l'entreprise & V. Multinomiale & $\begin{array}{l}\text { Inférieur à } 5 \text { ans } \\
\text { Entre 5 et } 10 \text { ans } \\
\text { Supérieur à 10 ans }\end{array}$ \\
\hline Structure de propriété & V. Multinomiale & $\begin{array}{l}\text { Entreprise familiale } \\
\text { Entreprise non familiale }\end{array}$ \\
\hline $\begin{array}{l}\text { Incertitude perçue de } \\
\text { l'environnement }\end{array}$ & V. Multinomiale & Mesurée par 9 items (échelle de Likert de 5 points) \\
\hline
\end{tabular}




\subsection{Les caractéristiques du terrain d'observation}

Nous entendons par PME toute entreprise juridiquement indépendante disposant d'un effectif compris entre 10 et 300 salariés (d'après la définition officielle de la PME donnée en 2006 par le conseil du marché financier tunisien). D'une manière très classique (Marchesnay, 1991), les critères retenus concernent d'une part le nombre de salariés et d'autre part l'indépendance de l'entreprise en termes de capital. L'effectif est un critère nécessaire mais insuffisant pour définir la PME. Les PME dépendantes d'entreprises plus grandes se voient en effet souvent proposer des procédures qui modifient leur système de gestion. A cet égard, Marchesnay (2003) explique par exemple que le développement des franchises contribue à renforcer la rigueur managériale dans la gestion des PME de distribution. Sont donc exclues de notre champ de recherche les très petites entreprises de moins de 10 salariés, les entreprises de plus de 300 salariés, ainsi que les filiales, succursales ou divisions d'entreprises plus importantes.

Nous avons réalisé cette étude auprès de PME tunisiennes. Les recherches en sciences de gestion en Tunisie sont rares, surtout en ce qui concerne les PME. Il est donc important de multiplier les recherches dans ce pays pour améliorer les connaissances relatives au système de gestion des dirigeants d'entreprises, et plus particulièrement, pour ce qui concerne notre étude, des dirigeants de PME.

Par ailleurs, le choix des PME industrielles se justifie par leur importance pour l'économie tunisienne. Composé de près de 6000 entreprises d'un effectif supérieur ou égal à 10 salariés, le tissu industriel tunisien est essentiellement constitué de PME qui emploient quelques 500000 personnes, assurant à elles seules $36 \%$ du PIB et $70 \%$ des exportations de biens du pays. Trois secteurs particulièrement développés dans les gouvernorats de Sousse, Monastir et Mahdia ont été ciblés : l'agroalimentaire, l'industrie lourde (construction métallique, traitements des métaux, équipements automobiles...) et l'industrie manufacturière (textile et habillement)

Les 101 PME interrogées se répartissent ainsi en fonction de leur secteur d'activité :

Tableau 2 : Répartition de l'échantillon en fonction du secteur d'activité

\begin{tabular}{|l|c|c|}
\hline \multicolumn{1}{|c|}{ Secteur d'activité } & Nombre & Pourcentage \\
\hline Entreprises agroalimentaires & 36 & $35,65 \%$ \\
\hline $\begin{array}{l}\text { Entreprises de l'industrie lourde (construction métallique, } \\
\text { traitements des métaux, équipements automobiles...) }\end{array}$ & 24 & $23,75 \%$ \\
\hline Entreprises manufacturières (entreprises de textile et d'habillement) & 41 & $40,60 \%$ \\
\hline \multicolumn{1}{|c|}{ Total } & $\mathbf{1 0 1}$ & $\mathbf{1 0 0 \%}$ \\
\hline
\end{tabular}

Parmi les 101 dirigeants interrogés, 26 (soit 25,75\%) ont précisé n'avoir effectué aucun changement dans leurs pratiques budgétaires au cours des cinq dernières années, et $75(74,25 \%)$ ont affirmé avoir innové en matière budgétaire au cours de cette même période. 
Les innovations observées portent en grande partie sur une augmentation significative de la diversité et/ou du degré de détail des budgets mis en place. Cependant, d'autres innovations, constituant des changements plus radicaux, ont également été observées dans un certain nombre d'entreprises. Il s'agit de l'instauration nouvelle d'un système budgétaire dans quatre cas de PME, de l'adoption de budgets par projet ou par activité dans trois cas, et de la mise en place d'un système budgétaire «base zéro » dans une des PME de l'échantillon. Ont également été observés dans cinq cas d'entreprises, un suivi bien plus soutenu des budgets et une implication accrue des dirigeants, constituant une nouvelle forme d'utilisation des budgets proche d'un mode de management interactif (Simons, 1995).

\subsection{La méthode d'analyse des données}

Le premier travail statistique a consisté à produire une typologie des 101 dirigeants de PME interrogés afin de pouvoir ensuite rapprocher les profils identifiés et l'adoption ou non d'innovations budgétaires.

Pour atteindre ce premier objectif, nous avons procédé en deux temps :

- dans un premier temps, une analyse en composante principale (ACP) s'appuyant sur les sept variables caractérisant les dirigeants a été établie. Ce traitement a permis de conserver pour l'analyse typologique les seules variables ayant une qualité de représentation acceptable.

- dans un second temps, une analyse typologique a été effectuée afin de faire émerger différents profils de dirigeants. Une classification ascendante non hiérarchique a été réalisée sur les 101 dirigeants de l'échantillon avec la méthode des nuées dynamiques. Cette méthode vise à constituer $k$ groupes à partir de $n$ individus de départ. Elle permet une convergence rapide, parfois même avec une simple itération, ce qui est très utile quand, tel est le cas dans notre étude, le volume de données est important et dépasse la centaine (Tufféry, 2006). Un partage de l'échantillon selon l'appartenance aux différents groupes identifiés par la méthode des nuées dynamiques a permis de repérer trois profils de dirigeants (45 dirigeants ont été classés dans la catégorie 1, 38 dans la catégorie 2 et 18 dans la catégorie 3). Des tentatives de classification en 2 et 4 groupes ont été réalisées mais les critères statistiques de validité ont montré que la classification en trois groupes était la plus pertinente.

Le second objectif de cette étude était de mettre en exergue l'existence d'un lien potentiel entre les facteurs de contingences retenus et l'adoption ou non d'innovations budgétaires au cours des cinq dernières années. Pour atteindre cet objectif, nous avons réalisé une analyse multivariée. Cette dernière permet de juger de l'effet des différentes variables prises dans leur ensemble. 
Prendre dans un même modèle toutes les variables de contingence donne une vision claire et plus réaliste des congruences entre les variables explicatives et la variable expliquée.

Etant donné l'objectif qui est de distinguer les PME innovantes en matière budgétaire de celles qui ne le sont pas, la variable à expliquer est dichotomique : nous utilisons la méthode du logit binaire. La variable dépendante qualitative Y prend la valeur « 1 » si l'entreprise i a effectué une innovation en matière budgétaire au cours des cinq dernières années $\left(\mathrm{y}_{\mathrm{i}}=1\right)$ et la valeur « 0 » sinon $\left(\mathrm{y}_{\mathrm{i}}=0\right)$. Pour Lemelin (2004, p.182), «cette fonction logistique est performante car relativement commode à manipuler et proche de la normale autour de la moyenne lorsque le nombre d'observations est important $\gg$.

\section{Résultats de la recherche}

Nous présentons tout d'abord les résultats relatifs à l'analyse typologique permettant d'identifier des profils de dirigeants. Nous présentons ensuite ceux du logit binaire permettant d'identifier les facteurs qui influencent l'adoption d'innovations budgétaires.

\subsection{Une typologie des dirigeants de PME tunisiens}

Une ACP a tout d'abord été réalisée afin de déterminer si les sept variables retenues pour caractériser le dirigeant influençaient significativement la construction de la typologie escomptée. Celle-ci nous a conduits à éliminer la variable «principal but du dirigeant » qui présentait une qualité de représentation médiocre car inférieure à 0,5 . Une seconde $\mathrm{ACP}$ a ensuite été lancée sur la base des six variables restantes.

Le test de sphéricité de Bartlett et le test MSA (mesure de précision de l'échantillon) de Kaiser, Meyer et Olkin (aussi appelé test KMO) ont été réalisés :

- Le test de sphéricité de Bartlett est «utilisé pour tester l'hypothèse nulle que les variables ne sont pas corrélées dans la population ... Une grande valeur du test favorise le rejet de l'hypothèse nulle, le test de Bartlett doit donc être significatif pour que les données soient factorisables » (Malhotra, 1993, p.621). Il montre, dans le cadre de notre étude, que l'analyse est significative avec un khi-deux très important $(203,873)$ et une $p$-value de $(p=0,000)$.

- Le test de KMO permet de tester l'adéquation de l'échantillon à l'analyse factorielle : « cet index compare les magnitudes des coefficients de corrélation observés à la magnitude des coefficients de corrélation partiels» (Malhotra, 1993, p.623). Nous obtenons un indice de KMO de 0,652 considéré par Kaiser et Rice $^{4}$ comme moyen mais acceptable.

\footnotetext{
${ }^{4}$ La calibration de la mesure de KMO par Kaiser et Rice est la suivante: MSA $>0.90$ : merveilleux; MSA $>0.80$ : méritoire; MSA>0.70 : moyen; MSA>0.60 : médiocre; MSA>0.50 : misérable; MSA $\leq 0.50$ : inacceptable (in Malhotra, 1993)
} 
Les données de l'ACP représentent ainsi un ensemble suffisamment cohérent pour qu'il soit raisonnable d'y rechercher des dimensions communes et réaliser une analyse factorielle (Evrard et al, 2009).

Tableau 3 : Résultats des deux tests mesurant la qualité de l'analyse factorielle

\begin{tabular}{|l|l|r|}
\hline \multirow{2}{*}{$\begin{array}{l}\text { Test de sphéricité de } \\
\text { Bartlett }\end{array}$} & Khi-deux approximé & 203,873 \\
\cline { 2 - 3 } & ddl & 45 \\
\cline { 2 - 3 } & $\begin{array}{l}\text { Signification de } \\
\text { Bartlett }\end{array}$ & $\mathbf{0 , 0 0 0}$ \\
\hline $\begin{array}{l}\text { Mesure de précision de l'échantillonnage de } \\
\text { Kaiser-Meyer-Olkin (KMO) }\end{array}$ & $\mathbf{0 , 6 5 2}$ \\
\hline
\end{tabular}

La méthode des nuées dynamiques a ensuite été utilisée pour mettre en évidence différents profils de dirigeants. Cette méthode est fortement recommandée lorsque le nombre d'observations dépasse la centaine, ce qui est le cas dans notre étude. Les tests réalisés montrent que le nombre de classes optimal est de trois (tableau 4):

Tableau 4 : Nombre d'observations dans chaque classe

\begin{tabular}{|c|c|c|}
\hline Classe & 1 & 45 \\
\cline { 2 - 3 } & 2 & 38 \\
\cline { 2 - 3 } & 3 & 18 \\
\hline \multicolumn{2}{|c|}{ Total } & $\mathbf{1 0 1}$ \\
\hline
\end{tabular}

Sur la base des six variables retenues, l'ACP et la classification réalisée selon la méthode des nuées dynamiques mettent donc en exergue l'existence, en Tunisie, de trois profils de dirigeants de PME contenant respectivement 45, 38 et 18 dirigeants :

\section{Groupe 1 : Les « jeunes gestionnaires »}

Ce groupe contient le nombre de dirigeants le plus important (45 soit 44,6\% d'entre eux). Plus de la moitié disposent d'un niveau de formation supérieur ou égal à la maîtrise. La majorité est titulaire d'un diplôme en gestion. Ils ont le plus souvent entre 35 et 45 ans et ont créé eux-mêmes leur PME. Culturellement, ces «jeunes gestionnaires » possèdent une forte aversion au risque et une attitude managériale orientée vers le long terme.

Il est à remarquer que ce profil de dirigeant est à l'opposé de celui dressé par Hofstede (2001) qui décrit le dirigeant arabe comme possédant une faible aversion au risque et une orientation court terme.

\section{Groupe 2 : Les « majeurs accomplis »}

Ce groupe rassemble 38 dirigeants (soit 37,6\% de l'échantillon). Il est composé de dirigeants ayant pour la plupart entre 45 et 65 ans et un niveau de formation qui ne dépasse pas le Bac+2. Leur type de formation (gestionnaire ou non gestionnaire) est en revanche très variable. Sur le 
plan culturel, les «majeurs accomplis » présentent une forte aversion au risque mais, contrairement aux «jeunes gestionnaires », adoptent une attitude managériale orientée vers le court terme.

\section{Groupe 3 : Les « patriarches expérimentés »}

Ce groupe rassemble un nombre moins important de dirigeants (18 soit 17,8\% de l'échantillon). Il est composé de dirigeants ayant, pour la plupart, entre 45 et 71 ans, disposant d'une formation de niveau peu élevé et de type non-gestionnaire. Ces «patriarches expérimentés » sont ceux qui s'apparentent le plus aux dimensions attribuées par Hofstede (2001) au monde arabe : ils sont faiblement averses au risque et orientés court terme.

Tableau 5 : Récapitulatif des trois groupes de dirigeants

\begin{tabular}{|l|l|l|l|}
\hline & \multicolumn{1}{|c|}{$\begin{array}{c}\text { Profil 1 « Jeunes } \\
\text { gestionnaires » }\end{array}$} & $\begin{array}{c}\text { Profil } 2 \text { « Majeurs } \\
\text { accomplis » }\end{array}$ & $\begin{array}{l}\text { Profil 3 « Patriarches } \\
\text { expérimentés » }\end{array}$ \\
\hline Age du dirigeant & $35-45$ ans & $45-65$ ans & 45- Plus de 65 ans \\
\hline Expérience du dirigeant & variable & Plus de 10 ans & Plus de 10 ans \\
\hline Niveau de formation & Bac+4 et plus & Bac +2 au plus & Moins que le Bac \\
\hline Type de formation & En sciences de gestion & variable & Non gestionnaire \\
\hline Aversion au risque & Forte aversion au risque & $\begin{array}{l}\text { Forte aversion au } \\
\text { risque }\end{array}$ & $\begin{array}{l}\text { Faible aversion au } \\
\text { risque }\end{array}$ \\
\hline $\begin{array}{l}\text { Orientation court terme } \\
\text { / long terme }\end{array}$ & Orientation long terme & $\begin{array}{l}\text { Orientation court } \\
\text { terme }\end{array}$ & Orientation court terme \\
\hline
\end{tabular}

4.2. L'émergence d'un lien entre secteurs d'activité, profils de dirigeants et adoption d'innovations budgétaires en PME

Les variables retenues dans notre étude pour expliquer l'adoption d'innovations budgétaires en PME sont le secteur d'activité et le profil des dirigeants. Nous avons également introduit au modèle des variables de contrôle pour affiner et enrichir le travail : la taille de l'entreprise, son âge, la structure de propriété (familiale ou non familiale) et l'incertitude perçue de l'environnement. Pour vérifier l'indépendance entre elles des variables du modèle, nous avons testé la corrélation entre celles-ci prises deux à deux avec un test de khi-deux. Ce test montre que ces variables sont indépendantes et peuvent être conservées pour l'élaboration du logit binaire. Nous avons ensuite vérifié l'absence de multicolinéarité entre ces mêmes variables en calculant le coefficient VIF (variance inflation factor). Bressoux (2008) indique que l'on peut parler de multicolinéarité lorsque le VIF dépasse 5. Les résultats présentés dans le tableau 6 montrent qu'aucune multicolinéarité n'existe entre les variables retenues dans notre étude puisque leur VIF est proche de 1 . 
Tableau 6 : Calculs de colinéarité

\begin{tabular}{|l|c|c|}
\hline \multirow{2}{*}{\multicolumn{1}{|c|}{ Modèle }} & \multicolumn{2}{c|}{ Statistiques de colinéarité } \\
\cline { 2 - 3 } & Tolérance & VIF \\
\hline Profils des dirigeants & 0,961 & 1,040 \\
\hline Secteur d'activité & 0,941 & 1,032 \\
\hline Structure de propriété & 0,926 & 1,080 \\
\hline Incertitude perçue de l'environnement & 0,981 & 1,019 \\
\hline Taille de la PME & 0,904 & 1,107 \\
\hline Age de la PME & 0,908 & 1,101 \\
\hline
\end{tabular}

Un test de la qualité du modèle de recherche a ensuite été réalisé. Un test du Khi-deux a permis de vérifier la significativité des variables : le log de vraisemblance est égal à 33,211.

Tableau 7 : La significativité du modèle

\begin{tabular}{|c|c|c|c|}
\hline & Khi-deux & Degré de liberté & Signification \\
\hline Etape & 33,211 & 19 & 0,023 \\
\hline Bloc & 33,211 & 19 & 0,023 \\
\hline Modèle & 33,211 & 19 & 0,023 \\
\hline
\end{tabular}

Ce ratio de vraisemblance mesure le pouvoir explicatif du modèle. Sa valeur, avec 19 degrés de liberté $(\mathrm{p}=0.023)$, est très significative. La combinaison des six variables retenues explique donc bien l'adoption ou non d'innovations budgétaires au cours des cinq dernières années dans les PME de l'échantillon. Le modèle de régression retenu est significatif.

Le lancement du logit binaire sur SPSS a donc pu être réalisé. Le tableau 8 attribue à chaque modalité de chaque variable trois valeurs :

- La valeur A qui constitue le coefficient estimé par la méthode du maximum de vraisemblance.

- $\quad$ Le degré de significativité de chaque facteur

- $\quad \operatorname{Exp}(B)$ qui précise le poids de l'effet d'une variable explicative sur la variable à expliquer.

Tableau 8 : Le logit binaire

\begin{tabular}{|c|c|c|c|}
\hline & A & Sig. & Exp(B) \\
\hline Jeunes gestionnaires & $\mathbf{2 , 0 6 2}$ & $\mathbf{0 , 0 0 7}$ & $\mathbf{7 , 8 6 3}$ \\
\hline Majeurs accomplis & $\mathbf{1 , 4 0 0}$ & $\mathbf{0 , 0 1 6}$ & $\mathbf{4 , 0 5 4}$ \\
\hline Patriarches expérimentés & 0,156 & 0,799 & 1,169 \\
\hline PME de l'agroalimentaire & $\mathbf{2 , 4 9 8}$ & $\mathbf{0 , 0 0 8}$ & $\mathbf{6 , 6 1 5}$ \\
\hline PME de l'industrie lourde & $-0,259$ & 0,719 & 0,772 \\
\hline PME manufacturières & $\mathbf{1 , 4 7 7}$ & $\mathbf{0 , 0 2 2}$ & $\mathbf{1 , 6 1 2}$ \\
\hline Entreprise familiale & 0,513 & 0,308 & 1,671 \\
\hline Environnement très incertain & $\mathbf{0 , 2 7 8}$ & $\mathbf{0 , 0 0 3}$ & $\mathbf{3 , 3 2 1}$ \\
\hline Environnement incertain & $\mathbf{0 , 2 9 7}$ & $\mathbf{0 , 0 1 2}$ & $\mathbf{1 , 8 4 5}$ \\
\hline Environnement partiellement certain & $-0,258$ & 0,750 & 0,772 \\
\hline Environnement certain & $-0,770$ & 0,364 & 0,463 \\
\hline Entre 10 et 50 employés & $-0,443$ & 0,422 & 0,642 \\
\hline Entre 51 et 100 employés & $-0,955$ & 0,146 & 0,385 \\
\hline PME âgée de moins 5 ans & $-1,495$ & 0,120 & 0,224 \\
\hline PME âgée de 5 à 10 ans & $-0,710$ & 0,236 & 0,492 \\
\hline Constante & 0,024 & 0,980 & 1,024 \\
\hline
\end{tabular}


Le tableau 8 montre que trois des variables introduites dans le modèle affichent une influence significative sur l'adoption d'innovations budgétaires :

- Le secteur d'activité influence significativement l'adoption d'innovations budgétaires dans les PME observées. Les PME du secteur agroalimentaire sont les plus innovantes $(\operatorname{Exp}(\mathrm{B})=6,615)$, les PME manufacturières le sont un peu moins $(\operatorname{Exp}(\mathrm{B})=1,612)$ et les PME du secteur de l'industrie lourde le sont rarement. L'adoption d'une innovation budgétaire est donc 6,615 fois plus probable dans une PME du secteur agroalimentaire et 1,612 fois plus probable dans une PME manufacturière que dans une PME moyenne. Notre étude montre ainsi la spécificité sectorielle de l'adoption d'innovations budgétaires en PME. Le secteur d'activité dans lequel opère la PME influence l'adoption d'innovations budgétaires.

- Le profil «jeunes gestionnaires » $(\operatorname{Exp}(\mathrm{B})=7,863)$ et, à un degré moindre, le profil «majeurs accomplis » $(\operatorname{Exp}(B)=4,054)$ influencent positivement l'adoption d'innovations budgétaires. Les «patriarches expérimentés » n’ont, le plus souvent, pas adopté d'innovations budgétaires au cours des cinq dernières années. L'adoption d'innovation budgétaire est donc 7,863 fois plus probable si le dirigeant est un «jeune gestionnaire » et 4,054 fois plus probable s'il est un «majeur accompli ». Tout comme le profil du dirigeant canadien (Lavigne, 2002), américain (Holmes et Nicholls, 1989), français (Chapellier, 2011), camerounais (Nyengue Edimo, 2006) ou syrien (Mohammed, 2010), le profil du dirigeant tunisien influence le système de gestion de la PME.

- Un environnement perçu comme très incertain ou incertain influence significativement et positivement l'adoption d'innovations budgétaires : la décision d'innover est 3,321 fois plus probable dans une PME évoluant dans un environnement perçu comme très incertain $(\operatorname{Exp}(B)=3,321)$ et 1,845 fois plus probable dans une PME évoluant dans un environnement perçu comme incertain $(\operatorname{Exp}(B)=1,845)$. Conformément aux résultats de Khandwalla (1972), Mintzberg (1982) ou Simons (1987), notre étude montre que l'incertitude perçue de l'environnement influence le système de gestion des dirigeants.

Aucune autre variable de contrôle n'est liée à l'adoption d'innovations budgétaires en PME.

\section{Discussion}

Nos résultats montrent que l'adoption d'innovations budgétaires dans les PME tunisiennes varie d'un secteur d'activité à l'autre, d'un contexte environnemental perçu à l'autre, et d'un profil de dirigeant à l'autre. Des spécificités sectorielles, contextuelles et individuelles sont ainsi mises en exergue : une majorité de dirigeants de PME a innové en matière budgétaire au cours des cinq dernières années, mais de manière différente selon le secteur d'activité dans lequel leur PME évolue, selon la manière dont ils perçoivent l'environnement, et selon leur profil. 
L'étude souligne tout d'abord une différenciation des pratiques selon le secteur d'activité. Nos travaux rejoignent sur ce point les conclusions d'Asselineau et Piré-Lechalard (2008) qui expliquent que des phénomènes de myopie ou d'orthodoxie sectorielle privent certaines organisations d'une perception de l'intérêt d'innover. Dans certains secteurs plus que dans d'autres, l'antagonisme peut s'avérer fort entre la logique de l'organisation générant des routines et des pratiques sociales, sources de stabilité et de réduction de l'incertitude, et la logique de l'innovation, supposant d'accepter incertitude et mouvement (Alter, 2000). Notre étude confirme aussi le constat relevé dans le manuel d'Oslo (2005) soulignant l'importance du rôle de l'Etat qui, grâce à une réglementation adaptée, un système fiscal incitatif ou des subventions ciblées, peut faciliter l'adoption d'innovations par les entreprises. Les PME du secteur agroalimentaire (identifiées comme les plus innovantes dans notre étude) sont en effet au cœur de la politique économique de l'Etat tunisien qui considère ce secteur en forte croissance comme stratégique pour le pays.

L'étude souligne ensuite une différenciation des pratiques selon que le dirigeant perçoive l'environnement dans lequel sa PME évolue comme certain ou incertain. Ce résultat concorde avec ceux de Dupuy (1994) qui relève des réactions réelles des systèmes comptables aux variations d'incertitude. Notre étude montre que les dirigeants de PME évoluant dans un environnement perçu comme incertain adoptent plus fréquemment des innovations budgétaires pour tenter de réduire cette incertitude. Ce résultat renforce les conclusions de Germain (2004), Haldma et Lääts (2002) et Abdel-Kader et Luther (2008) qui affirment que les dirigeants de PME évoluant dans un environnement perçu comme incertain disposent d'un système de contrôle plus complexe et plus formalisé que ceux évoluant dans un environnement perçu comme simple et stable.

Cette étude confirme enfin que si certaines caractéristiques structurelles et contextuelles sont effectivement liées à l'adoption d'innovations budgétaires en PME, le dirigeant tient un rôle très important, celui-ci étant au cœur de toute démarche de changement au sein de son entreprise. Sa perception et ses comportements facilitent ou au contraire compromettent l'émergence d'innovations (Agro et al, 1996). Dans l'étude ici présentée, certains profils de dirigeants (les jeunes gestionnaires) facilitent l'adoption d'innovations budgétaires. Cette observation peut être interprétée comme le fruit de pratiques isomorphes, voire mimétiques, liées à la formation poussant ces dirigeants à l'adoption des pratiques budgétaires innovantes inculquées dans les mêmes cursus de gestion qu'ils ont suivis. D'autres profils, tels que les patriarches expérimentés, constituent un frein à l'innovation. Ce résultat rejoint celui de Saint-Pierre et Delisle (2006) qui 
expliquent que les dirigeants de PME privilégient les outils dont ils estiment avoir besoin. Or, ces besoins varient d'un dirigeant à l'autre.

Deux conséquences managériales peuvent à notre sens être tirées de ces résultats.

La diversité des innovations budgétaires en réponse à la diversité des situations observées nous conduit tout d'abord à penser, comme Oriot et Misiaszek (2012, p.39) que «c'est aux systèmes de management de la performance de s'adapter aux réalités des différentes $P M E$ et non à ces dernières de passer sous les fourches caudines de modèles prédéfinis quels qu'ils soient ». A l'instar des travaux de Malerba (2005), notre étude montre ainsi que la diversité des contextes et des acteurs oblige les institutions, organisations et réseaux composant les systèmes sectoriels de l'innovation (SSI) à tenir compte des spécificités sectorielles et individuelles pour favoriser la production de connaissances nouvelles utiles à l'innovation en PME. Cette condition semble nécessaire pour que ces institutions, organisations et réseaux contribuent à favoriser la production de connaissances nouvelles et l'innovation (Malerba, 2004). Il ne s'agit donc pas pour les SSI d'apporter aux dirigeants des solutions «toutes prêtes » conformes à ce que Marchesnay (2003) appelle «des préceptes managériaux» supposés universellement applicables. Il ne s'agit pas d'imposer aux dirigeants un point de vue normatif mais de leur proposer une aide et des solutions spécifiques adaptées aux secteurs, aux contextes et aux profils qui sont eux-mêmes spécifiques. L'objectif est de parvenir à ce que le dirigeant puisse interpréter et sélectionner les connaissances et les conseils avec discernement et intelligence. Les experts des institutions, organisations et réseaux composant les systèmes sectoriels de l'innovation (SSI) doivent en quelque sorte jouer le rôle du jardinier: faire en sorte, avec patience et engagement, que la «greffe de l'innovation managériale » puisse prendre. Ainsi, une personnalisation de la contribution et une pédagogie adaptée seront décisives.

Mais l'article montre aussi que les «jeunes gestionnaires », dirigeants aux perceptions et profils identiques, sont les plus enclins à adopter des innovations budgétaires. Aussi, au-delà de l'approche en termes de contingence qui vient d'être développée, une autre interprétation, fondée sur une lecture néo-institutionnelle de ce résultat peut apporter un éclairage pertinent. On peut en effet supposer, à l'instar des théoriciens néo-institutionnels (Meyer et Rowan, 1977 ; Di-Maggio et Powell, 1983), que l'adoption d'innovations budgétaires par les « jeunes gestionnaires » soit le fruit de comportements isomorphiques. Les «règles» et «codes» acquis au cours de leur formation à la gestion pourraient en effet expliquer leur tendance à adopter les mêmes pratiques, analysé dans ce cas comme un isomorphisme de type normatif. Par ailleurs, suivant cette approche néo-institutionnelle, l'incertitude perçue de l'environnement pourrait être analysée, audelà de toute interprétation contingente, comme un facteur de renforcement de l'isomorphisme, 
mimétique notamment, conduisant certaines organisations à dupliquer les actions menées par les autres. La théorie institutionnelle souligne en effet qu'en situation d'incertitude les entreprises ont tendance à copier et reproduire les pratiques de leurs concurrents, considérés alors comme « les bons élèves ».

\section{Conclusion}

Le profil des dirigeants, le secteur d'activité, et l'incertitude perçue de l'environnement apparaissent dans notre étude comme des variables clés liées à l'adoption d'innovations budgétaires en PME.

Ce résultat montre tout d'abord que des dirigeants aux perceptions et profils différents, évoluant dans des secteurs et des environnements différents, adoptent et usent d'outils de gestion différents. A l'instar de Lorino (2013), nous pensons que la diversité des contextes et des acteurs conduit à ne pas trop placer d'espoir dans une pensée normative qui définirait l'existence de solutions budgétaires standards susceptibles de s'imposer à tous les acteurs, de toutes les organisations. Mettre en œuvre des outils de gestion standards non adaptés, qui véhiculeraient implicitement la référence à une rationalité plus ou moins substantielle, voire normative, ne ferait pas sens pour les dirigeants de PME. Oriot et Misiaszek (2012) montrent par exemple qu'une innovation managériale telle que le Balanced Scorecard, de par son caractère normatif, n'est pas adapté aux PME, en particulier en contexte de redressement. La diversité des contextes et des acteurs obligent ainsi les institutions, organisations et réseaux composant les systèmes sectoriels de l'innovation (SSI) à tenir compte de ces attentes et à adapter leur contribution. Cette condition est nécessaire pour que l'action planifiée et déployée par l'ensemble des agents qui interagissent à l'intérieur d'un secteur pour favoriser l'innovation, soit efficace. Nous sommes ici dans le monde de la diversité et de la singularité.

Par ailleurs, ce travail montre également que des dirigeants aux perceptions et profils identiques adoptent des pratiques innovantes identiques. Cette observation peut être interprétée comme le fruit de pratiques isomorphes, voire mimétiques, liées à la fois à un environnement incertain conduisant les PME à «se mimer » les unes les autres, et à la formation des dirigeants poussant à l'adoption de pratiques identiques innovantes inculquées dans les universités ou dans les écoles de commerce.

Cette recherche n'est pas exempte de limites. Elles sont tout d'abord liées à la taille de l'échantillon : la constitution d'un échantillon de 101 observations directes en face-à-face constitue un travail important pour le chercheur mais le statisticien le trouvera trop limité. Les circonstances politiques et sécuritaires de la Tunisie au moment du recueil des données nous ont obligés à limiter ce nombre. Ensuite, pour des raisons de praticabilité, certains facteurs de 
contingence susceptibles d'influencer l'adoption d'innovations budgétaires en PME n'ont pu être pris en compte ; ainsi les aspects psychologiques de la personnalité du dirigeant comme son attitude face au changement (Smith, 1967 ; Julien et Marchesnay, 1996), son esprit d'innovation (Smith, 1967 ; Miles et Snow, 1978) ou son style de management (Holmes et Zimmer, 1994), n'ont pas été retenus dans notre étude. Notons enfin que seules des PME industrielles de trois secteurs différents ont été intégrées à l'échantillon. Les PME d'autres secteurs industriels, de services et commerciales n'ont pas été associées à cette étude. Les résultats obtenus ne peuvent donc en aucun cas être généralisés à l'ensemble des secteurs de la vie économique tunisienne.

Notre ambition est de poursuivre cette recherche en la dupliquant dans d'autres secteurs d'activité (notamment dans des PME commerciales et de services) et dans d'autres pays (en premier lieu en France) en vue de mener des analyses comparatives relatives aux facteurs influençant l'adoption d'innovations budgétaires. Une étude qualitative sous forme d'entretiens et d'études de cas permettrait par ailleurs d'obtenir une représentation plus fine et une analyse plus approfondie des phénomènes observés. Elle permettrait tout d'abord d'approfondir les perspectives de recherche intéressantes ouvertes par l'analyse institutionnelle sur les questions liées à l'adoption isomorphique des innovations en PME. Elle permettrait ensuite de mieux comprendre pourquoi et comment des dirigeants de PME finalement assez éloignés du cliché du «petit patron » soi-disant averse à l'usage de nouveaux outils de gestion formalisés, innovent pour améliorer les techniques et les méthodes formelles de gestion existantes.

\section{Bibliographie}

ABDEL KADER M., LUTHER R. (2008), « The impact of firm characteristics on management accounting practices: A UK based empirical analysis », The British Accounting Review, vol. 40, n¹, p.227.

ABERNATHY, W. J., UTTERBACK J.M. (1978), "Patterns of innovation in technology", Technology review, $80: 7,40-47$.

AFFES H., CHABCHOUB A. (2007), «Le système d'information comptable : les déterminants de ses caractéristiques et son impact sur la performance financière des PME en Tunisie », Revue des Sciences de Gestion, Direction et Gestion, N $^{\circ} 224-225$, p 59-68.

AGRO L., CORNET A., PICHAULT F. (1996), « Système d'information : quelle implication pour les utilisateurs ? », Revue Française de Gestion, N 110 , sept-oct, p. 46-55.

ALCOUFFE S., (2006), «La recherche sur les innovations managériales en comptabilité et contrôle de gestion : proposition d'un modèle théorique », in l'appropriation des outils de gestion : vers de nouvelles perspectives théoriques?, Coordonné par A. Grimand, Université de Saint-Etienne

ALCOUFFE S., BERLAND N., LEVANT Y. (2003), "Les facteurs de diffusion des innovations managériales en comptabilité et contrôle de gestion: une étude comparative", Comptabilité-ContrôleAudit, Numéro spécial, p. 7-26

ALCOUFFE S., BERLAND N., LEVANT Y. (2008), " "Succès" et "échec" d'un outil de gestion. Le cas de la naissance des budgets et de la gestion sans budget ", Revue Française de Gestion, Vol. 34, n¹88189, p291- 306.

ALTER N., (2000), L'innovation ordinaire, Paris, Presses Universitaires de France.

ASSELINEAU A., PIRE-LECHALARD P., (2008), « Développement durable et entreprise responsable : une voie pour l'innovation de rupture? », $3^{\text {ème }}$ Journée Neptune, Toulon 
BALDRIDGE, J.V., BURNHAM, R.A. (1975), « Organizational innovation : individual, organizational and environmental impacts », Administrative Science Quarterly, n ${ }^{\circ} 20$, p165-176.

BANHAM R., (2000), « Better Budgets », Journal of Accountancy, feb: 7-11.

BERGERON, H. (1996), Différenciation des systèmes de données et représentations en contrôle de gestion : essai d'observation et d'interprétation, Thèse de doctorat, Univ. Montpellier II

BERLAND N. (1999), L'histoire du contrôle budgétaire en France. Les fonctions du contrôle budgétaire, influences de l'idéologie, de l'environnement et du management stratégique, Thèse de doctorat, Université ParisIX-Dauphine

BERLAND N., (2002), Le contrôle budgétaire, Repères, La Découverte

BERLAND N. (2004), « La gestion sans budget : évaluation de la pertinence des critiques et interprétation théorique », Finance Contrôle Stratégie, 7(4), déc: 37-58.

BERLAND N., CHIAPELLO E., (2009), "Criticisms of capitalism, budgeting and the double enrolment: Budgetary control rhetoric and social reform in France in the 1930s and 1950s", Accounting, Organizations and Society 34(1): 28-57.

BESCOS P.L, MENDOZA C. (1998), «Les besoins d'information des managers sont-ils satisfaits », Revue Française de Gestion, novembre-décembre, p.117-128.

BESCOS, P. L., CAUVIN E., LANGEVIN P., MENDOZA C. (2004), «Critiques du budget : une approche contingente », Comptabilité Contrôle Audit, vol. 10, $\mathrm{n}^{\circ}$ 1, p. 165-185.

BIRKINSHAW J., HAMEL G., MOL M.J., (2008), «Management Innovation », Academy of Management Review, vol. 33, No. 33825 - 845

BOLDRINI J.P., (2008), «Economie et gestion de la petite et moyenne entreprise », Revue internationale $P M E, \mathrm{Vol}) 21, \mathrm{~N}^{\circ} 1, \mathrm{p} 9-34$

BOUQUIN H. (1998), Le contrôle de gestion, PUF, $5^{\text {ème }}$ éd

BRESSOUX P. (2008), Modélisation statistique appliquée en sciences sociales, Ed. De Boeck, Bruxelles.

BRIMSON J.A, ANTOS J., (1999) Driving value using Activity-based-budgeting, John Wiley, Inc.

BRION S., MOTHE C., SABATIER M. (2008), «L'impact-clé des modes de management pour l'innovation », Revue Française de Gestion, n ${ }^{187}$, octobre, 177-194.

CHAPELLIER P (1996). «Données comptables de gestion et système d'information du dirigeant de PME », Système d'Information et Management 2 (1): 23-41.

CHAPELLIER P., (2011), Vers un modèle de gestion hybride pour le dirigeant de PME : Une étude de la triangulation entre système d'information formel, recours à l'expert et mètis du dirigeant, HDR - Univ. Nice Sophia Antipolis

CHAPELLIER P., DUPUY Y. (2013), «L'hybridation du système de gestion du dirigeant de la PME pérenne » in «Pilotage de la pérennité organisationnelle : normes, représentations et contrôle » coordonné par S. Mignon, avril, Ed EMS

CHEFFI, W., NEKHILI M. (2011). « Rôles assignés à la comptabilité de gestion par les managers et changement comptable : question d'adéquation ou simple désillusion? », Comptabilité, Contrôle, Audit, tome 17, Vol.1, p.67-97.

CHONG, V.K, CHONG K.M. (1997), «Strategic choices, environmental uncertainty and SBU performance: a note on the intervening role of management accounting systems », Accounting and Business Research, vol. 27, ${ }^{\circ}$ 4, p. 268-276.

COOPER R., SLAGMULDER, R. (2000), Activity- based budgeting. Strategic Finance part 1

CROZIER M., FRIEDBERG E. (1977), L'acteur et le système, Paris, Edition du Seuil.

DAMANPOUR F., ARAVID D., (2011), «Managerial innovation: Conceptions, processes and antecedents », Management and Organization Review, $\mathrm{Vol}^{\circ} 8, \mathrm{~N}^{\circ} 2$, p 423-454

DAMANPOUR F., SCHNEIDER M., (2006), « Phases of the adoption of innovation in organizations: Effects of environment, organization, and top managers », British Journal of Management, $\mathrm{Vol}^{\circ} 17, \mathrm{~N}^{\circ} 3, \mathrm{p}$ 215-236.

DESMAZES J., LAFONTAINE J.P. (2007), «L'assimilation des budgets environnementaux et du tableau de bord vert par les entreprises ». Congrès de l'AFC, Poitiers.

DFCG (1994), Les Directeurs financiers et la procédure budgétaire - Faut-il tuer le budget ?, DFCGKPMG, IFOP

DHIFALLAH S., CHANAL V., DEFELIX C. (2008), «Quelle gestion des ressources humaines dans les organisations ambidextres ? », Revue Française de Gestion, $n^{\circ}$ 187, p 161-175 
DI MAGGIO P.J., POWELL W.W. (1983), "The iron cage revisited: Institutional isomorphism and collective rationality in organizational fields", American Sociological Review, 48, p147-160

DUPUY Y., KALIKA M., MARMUSE C., TRAHAND, J. (1989), Les systèmes de gestion, introduction au soft management, Paris, Vuibert.

DUPUY Y. (1994), «Une lecture des recherches actuelles en comptabilité-contrôle », XII ${ }^{\text {èmes }}$ Journées Nationales des IAE, Montpellier février 1994, p.17-33.

DUPUY Y., VILLESEQUE F. (2003), «Vers des budgets transversaux ? », Echanges, ${ }^{\circ} 205$

EVRARD Y, PRAS B, ROUX E, (2009), « Market - Fondements et méthodes des recherches en marketing », $4^{\text {ème }}$ Ed Dunod

FERRARY M. (2008), «L'innovation radicale : entre cluster ambidextre et organisations spécialisées », Revue Française de Gestion, $\mathrm{n}^{\circ} 187, \mathrm{p} 109-125$

FONROUGE C. (2002), «L'entrepreneur et son entreprise : une relation dialogique » Revue française de gestion, $\mathrm{n}^{\circ} 138$, avril-mai, p. 145-1582.

GADILLE M., D'IRIBARNE A. (2000), «La diffusion d'Internet dans les PME. Motifs d'adoption dans les réseaux et ressources mobilisées », Réseaux, vol 18, n¹04, pp. 59-92.

GERMAIN C. (2001), "Le pilotage de la performance dans les PME, les résultats d'une enquête empirique », $22^{\text {ème }}$ congrès de l'Association Francophone de Comptabilité, Metz

GERMAIN C. (2004), «La contingence des systèmes de mesure de la performance : les résultats d'une recherche empirique sur le secteur des PME », Finance, Contrôle, Stratégie, Vol ${ }^{\circ}$, N ${ }^{\circ} 1$, p33-52.

GODOWSKI C. (2003), «Essai sur la dynamique d'assimilation des innovations managériales : le cas des approches par activité », Comptabilité-Contrôle-Audit, $\mathrm{n}^{\circ}$ spécial, p71-86

GOVINDARAJAN V., TRIMBLE C., (2012), Reverse Innovation: Create Far From Home, Win Everywhere, Harvard Business Review Press

HALDMA T., LÄÄTS K. (2002), «Contingencies influencing the management accounting practices of Estonian manufacturing companies », Management Accounting Research, vol. 13, n 4, p379-400.

HAMEL G. (2006), "The why, what and how of management innovation", Harvard Business Review, p.74-84.

HAMEL G. (2007) The Future of Management, Harvard Business School Press

HAUSMAN A. (2005), «Innovativeness among small businesses : theory and propositions for future research », Industrial Marketing Management, vol. 34, p. 773-782.

HENDERSON R.M., CLARK, K.B. (1990), «Architectural innovations : the reconfiguration of existing product technologies and the failure of established firms », Admnistrative science quaterly, $\mathrm{Vol}^{\circ} 14, \mathrm{p} .375$ 397.

HOFSTEDE G. (2001), Culture's Consequences: comparing values, behaviors, institutions, and organizations across nations ( $2^{\text {nd }}$ ed.), Thousand Oaks, CA: SAGE Publications, p.9.

HOLMES S., NICHOLLS D. (1989), « Modelling the accounting information requirement of small business », Accounting and Business Research, vol. 19, $\mathrm{n}^{\circ}$ 74, p.143-150.

HOLMES, S., ZIMMER, I. (1994), 'The nature of the small firm: understanding the motivations of growth and non-growth oriented owners', Australian Journal of Management, vol. 19, no. 1, pp. 97-120.

HOPE J, FRASER R, (1997), "Beyond budgeting, Breaking through the barrier to 'the third wave », Management Accounting, Déc, p 20-23.

HOPE J., FRASER R (1999), "Beyond Budgeting...building a new management model for the information age", Management Accounting, :16-21.

HUET F. (2006) "Capacités d'innovation et coopération de PME : des effets auto-renforçants". Revue Internationale des PME, Vol.19, $\mathrm{N}^{\circ} 1, \mathrm{p} 95-101$

JAOUEN A, LE ROY F. (2013) L'innovation managériale, Collection: Management Sup, Dunod

JORDAN H. : (1998), Planification et contrôle de gestion en France en 1998, Groupe HEC

JULIEN PA. (1997), «Pour une définition des PME». in Julien PA : Les PME : Bilan et perspectives. Economica.

JULIEN P.A., MARCHESNAY M. (1996), L'Entrepreneuriat, Paris, Economica.

KESSLER, E.H., CHAKRABARTI, A.K., (1996), «Innovation speed: a conceptual model of context, antecedents and outcomes ». Academy of Management Review, $\mathrm{N}^{\circ} 21$, p1143-1191.

KHAN A. M., MANOPICHETWATTANA V. (1989a), «Innovative And Noninnovative Small Firms: Types And Charact », Management Science, vol. 35, nº 5, p. 597-606. 
KHAN A. M., MANOPICHETWATTANA V. (1989b), « Models for Distinguishing Innovative and Noninnovative Small Firms », Journal of Business Venturing, vol. 4, nº 3, p. 187-196.

KHANDWALLA P.N, (1972), "The effects different types of competition on use managerial controls", Journal Accounting Research, vol. 10, n 2, p. 275-285

KOTEY B., MEREDITH G., (1997), "Relationship among owner/manager personal values, business strategies and enterprise performance”, Journal of Small Business Management, $\mathrm{Vol}^{\circ} 32, \mathrm{~N}^{\circ} 2$, p37

LAVIGNE B. (2002), « Contribution à l'étude de la genèse des états financiers des PME », Comptabilité, Contrôle, Audit, Tome 8, Vol 1, mai, p.25-44.

LAVIGNE B., SAINT-PIERRE J. (2002). "Association entre le système d'information comptable des PME et leur performance financière ». $6^{\text {ème }}$ Congrès International Francophone sur la PME (CIFPME), Montréal (Québec)

LEMELIN A. (2004), « Méthodes quantitatives des sciences sociales appliquées aux études urbaines et régionales ». Édition révisée. Montréal : INRS-UCS

LE ROY F., ROBERT M., GIULIANI P. (2012) «Quels liens entre l'innovation technologique et managériale ? Pour une distinction entre l'innovation produit et l'innovation procédé », $21^{\text {ème }}$ conférence de l'AIMS, Lille

LORINO P. (1995), Comptes et récits de la performance, Editions d'Organisation.

LORINO P. (2013), préface de Pilotage de la pérennité organisationnelle : normes, représentations et contrôle, coordonné par S. MIGNON, Ed EMS

MALERBA, F. (2004), Sectoral Systems of Innovation: Concept, Issues and Analysis of Six Major Sectors in Europe,.Cambridge University Press.

MALERBA, F. (2005), “Sectoral Systems: How and why innovation differs across sectors." In Fagerberg, David and Nelson (eds.) The Oxford Handbook of Innovation, Oxford University Press; p308-406

MALHOTRA N. K, (1993), Marketing research: An applied orientation, Englewood Cliffs, NJ, Prentice Hall.

MANUEL D’OSLO (2005), Principes directeurs pour le recueil et l'interprétation des données sur l'innovation, $3^{\text {ème }}$ édition, OCDE

MARCHESNAY, M. (1991), «La P.M.E. : une gestion spécifique », Économie Rurale, N²06, p11-17.

MARCHESNAY M (1997). La spécificité de la gestion des PME, in Petites entreprises et grands enjeux, le développement agroalimentaire local, L'Harmattan.

MARCHESNAY M. (2003). «La petite entreprise : sortir de l'ignorance », Revue Française de Gestion, Vol 29, n¹44, mai-juin, p.107-118.

MARTINEAU R. (2008), "Les outils de gestion, lieu de rencontre entre théorie et pratique : une revue de littérature », Congrès des IAE, septembre, Lille

MEYER J.W., ROWAN B. (1977), "Institutional organizations: formal structure as myth and ceremony", American Journal of Sociology, 83: 340-363.

MILES R. E., SNOW C.C. (1978), «Organizational strategy, structure, and process », McGraw-Hill, New York

MINTZBERG H. (1982). Structure et dynamique des organisations, Les Éditions d'Organisation.

MOHAMMED A. (2010), Etude du système d'information comptable des PME syriennes : observation et essai de compréhension des pratiques, Thèse de doctorat, Université de Nice Sophia Antipolis

MOISDON J.C. (1997), Du Mode d'Existence des Outils de Gestion, Ed. Seli Arslan, Paris.

MOL M. J., BIRKINSHAW J. (2006), "Against the flow: reaping the rewards of management innovation", European Business Forum, issue 27: 24-29

MOL M.J, BIRKINSHAW J. (2009), «The sources of management innovation: When firms introduce new management practices », Journal of Business Research, $\mathrm{Vol}^{\circ} 62$, p. 1269-1280.

MOUSLI M. (2007), L'efficience du système budgétaire comme conséquence du style de management stratégique des directions générales : cas de sociétés cotées françaises. Thèse de doctorat, Lyon 3.

NARO G. (2006). "Un tableau de bord stratégique pour les dirigeants de petites entreprises :apports et limites des tableaux de bord prospectifs », Fourcade C., Paché G., Pérez R. (éds).

NGUYEN TAN HON V. (2008), Débats et conflits lors de l'élaboration du budget, Thèse de doctorat, HEC

NOBRE T. (2001), «Méthodes et outils du contrôle de gestion dans les PME », Finance, Contrôle, Stratégie, vol. 4, n², p.119-148. 
NOBRE T., BIRON J. (2002), «L'ABC à l'hôpital : le cas de la chirurgie infantile », Finance Contrôle Stratégie, vol 5, $\mathrm{n}^{\circ}$, juin, pp. 85-105.

NYENGUE EDIMO P. (2006), L'organisation du système d'information comptable des entreprises camerounaises : essai d'observation et interprétation des pratiques. Thèse de doctorat, Univ. Bordeaux 4 ORIOT F., MISIASZEK E., (2012), «Le Balanced Scorecard au filtre d'une PME française ou pourquoi les PME préfèrent le « sur-mesure » », Revue française de gestion, $\mathrm{Vol}^{\circ}{ }^{\circ}$, $\mathrm{N}^{\circ} 225$, p.27-43.

PYRRH P.A., (1976), «Et si vos budgets partaient de zéro ? » Revue Harvard l'Expansion, n²

RAYMOND L., BLILI S., EL-ALAMI D. (2004), «L'écart entre le consultant et la PME : analyse et perspectives », Gestion, vol. 28, n 4, p.52-60.

SABOLY M. (1994). Les déterminants de la qualité des produits comptables des entreprises : Le rôle du dirigeant, Thèse de sciences de gestion, Poitiers.

SAINT-PIERRE J., DELISLE S. (2006). "An expert diagnosis system for the benchmarking of SMEs' performance”, Benchmarking: An International Journal, Vol.13, ํ1-2, p.106-119

SALEH, S.D., WANG, C.K. (1993), «The management of innovation - strategy, structure, and organizational climate » Transactions on Engineering Management, $\mathrm{N}^{\circ} 40, \mathrm{p} 14-21$

SCHMIDT J.A. (1992), "Is it time to replace traditional budgeting ?", Journal of Accountancy, oct., 103107

SHIELDS J., YOUNG M. (1993), « Antecedents and consequences of participative budgeting: evidence on the effects of asymmetrical information », Journal of Management Accounting Research, $\operatorname{Vol}^{\circ} 5, \mathrm{~N}^{\circ} 1, \mathrm{p}$ 265-280

SIMON F., TELLIER A. (2008), «Créativité et réseaux sociaux dans l'organisation ambidextre », Revue Française de Gestion, n ${ }^{\circ}$ 187, 2008/7, p145-159

SIMONS R. (1987), «Accounting control systems and business strategy : an empirical analysis », Accounting, Organizations and Society, Vol.12, n 4, pp.357-374.

SIMONS R. (1995), Levers of Control, Boston, Harvard Business School Press.

SMITH N.R. (1967). «The Entrepreneur and His Firm: The Relationship between Type of Man and Type of Company », Bureau of Business and Economic Research Michigan

SOLLE G., ROUBY E. (2003), « De la conception des innovations managériales en contrôle de gestion : Quelles propositions ? », Comptabilité-Contrôle-Audit, $\mathrm{N}^{\circ}$ spécial, p 147-168.

SPONEM S. (2004), Diversité des pratiques budgétaires des entreprises françaises: proposition d'une typologie et analyse des déterminants, Thèse de doctorat, Univ.Paris IX

TEECE D. J., PISANO G., SHUEN A. (1997) "Dynamic capabilities and strategic management", Strategic Management Journal, 18 : 7, 509-533.

THIETART R.A. (2007). Méthode de recherche en management, $3^{\text {ème }}$ éd, Dunod

TUFFERY S. (2006). «Data mining et statistiques décisionnelles », Ed technip, Paris

VILLESEQUE F., (2003), « Transversalité et Systèmes budgétaires, un essai d'observation et d'analyse » , Thèse de doctorat, Univ. Montpellier 2

VILLESEQUE-DUBUS F. (2005), «Vers une transversalisation des budgets : un essai d'observation et d'interprétation », Comptabilité, Contrôle Audit, tome 11, vol.2, p127-148

WALLEY P., BLENKINSOP S., DUBERLEY J. (1994) « The adoption and non-adoption of modern accounting practices: A study of 20 manufacturing firms », International Journal of Production Economics, Vol.36, $\mathrm{N}^{\circ} 1, \mathrm{p} 19-27$

WEST M.A., (1990), « The social psychology of innovation in groups », In West, M.A. and Farr, J.L. (eds), Innovation and Creativity at Work: Psychological and Organizational Strategies. Chichester, UK:John Wiley, pp. 309-333.

ZAHRA S. A., NEUBAUM D.O., HUSE M., (2000), "Entrepreneurship in medium-size companies:Exploring the effects of ownership and governance systems", Journal of Management, $N^{\circ} 26$, p947-976.

ZMUD R., LINK A. (1984) "Firm Size and Innovation Activity in the Video Display Terminal Industry", in B. Bozeman, A. Link and M. Crow (Eds.), Strategic Management of Industrial R\&D, D.C. Heath, Lexington 